\title{
The prevalence of malnutrition in hemodialysis patients
}

\author{
Ali Ghorbani ${ }^{1}$, Fatemeh Hayati ${ }^{1}$, Majid Karandish ${ }^{2}$, Soudabeh Sabzali ${ }^{1,2^{*}}$ \\ ${ }^{1}$ Department of Internal Medicine, School of Medicine, Chronic Renal Failure Research Center and \\ ${ }^{2}$ Department of Nutritional Sciences, School of Paramedical Sciences, Diabetes Research Center, Jundishapur University of Medical \\ Sciences, Ahvaz, Iran
}

\section{A R T I CLE IN F O}

\section{Article Type:}

Original

\section{Article History:}

Received: 10 August 2019

Accepted: 20 December 2019

Published online: 17 Jnauary 2020

\section{Keywords:}

Hemodialysis

Malnutrition

Nutrition assessment

End-stage renal disease

Chronic kidney disease

Protein energy wasting

Subjective global assessment

\begin{abstract}
A B S T R A C T
Introduction: Malnutrition is a complex, multi-factorial and common problem in patients with end-stage renal disease (ESRD) undergoing hemodialysis that increases morbidity and mortality rate in them.

Objectives: To assess the prevalence of malnutrition and associated factors in hemodialysis patients.

Patients and Methods: This cross-sectional study was conducted on 239 hemodialysis patients (162 males and 77 females) referred to three dialysis centers in Ahvaz, Iran in 2018. The nutritional status was measured using subjective global assessment (SGA) tool. The anthropometric indices, body mass index (BMI) and biochemical parameters including albumin, triglyceride, C-reactive protein (CRP), and adequacy of dialysis (Kt/V) were also measured in all patients.

Results: Based on SGA, 45 (18.8\%) patients including 32 males and 13 females had mild to moderate malnutrition while $26(10.9 \%)$ patients consisting of 11 men and 15 women had severe malnutrition. We found a significant association between patients' gender and malnutrition status $(P=0.013)$. In addition, a significant association was seen between age $(P=0.024)$, BMI $(P=0.0001)$, CRP $(P=0.047)$ and serum albumin concentration $(P=0.007)$ with nutritional status. However, we did not find any significant association between triglyceride, Kt/V and duration of hemodialysis per week with malnutrition $(P>0.05)$. Moreover, BMI, patient's gender and serum albumin were the significant predictors of severe malnutrition in hemodialysis patients $(P<0.05)$.

Conclusion: According to high prevalence of malnutrition in ESRD patients undergoing hemodialysis, periodic assessment of nutritional status is necessary for them. Moreover, female patients, CRP, serum albumin and BMI showed a significant correlation with SGA score, which are important to determine the nutritional status of hemodialysis patients (HD) patients.
\end{abstract}

Implication for health policy/practice/research/medical education:

In a study on 239 hemodialysis patients (Ahvaz, Iran), we found female patients, C-reactive protein, serum albumin and body mass index had a significant correlation with nutritional status.

Please cite this paper as: Ghorbani A, Hayati F, Karandish M, Sabzali S. The prevalence of malnutrition in hemodialysis patients. J Renal Inj Prev. 2020; 9(2): e15. doi: 10.34172/jrip.2020.15.

\section{Introduction}

Chronic kidney disease (CKD) is a global widespread epidemic disease, which its prevalence rate is $5-15 \%$. The incidence rate of end-stage renal disease (ESRD) patients requiring dialysis is also increasing $(1,2)$. Hemodialysis patients have a much higher mortality rate than the general population $(3,4)$. Several risk factors contribute to increased mortality rate in hemodialysis patients. The most important risk factor is cardiovascular disease $(5,6)$. An important non-cardiovascular risk factor of mortality among hemodialysis patients is malnutrition (7).

American Society for Parenteral and Enteral Nutrition has defined malnutrition as "an imbalance of need and supply of energy, protein and micronutrients leading to growth and development defect" (8). Malnutrition in the form of protein energy wasting (PEW) is highly prevalent in hemodialysis patients (9).

Inadequate intake of certain nutrients, anorexia, nutrient losses during hemodialysis, and elevated protein catabolism due to increased production of inflammatory cytokines and drug-related factors contribute to nutritional deficits in hemodialysis patients (10).

Nutritional inadequacy caused by numerous factors (e.g. metabolic acidosis, altered intestinal flora, and 
hormonal dysregulation) can progress kidney disease and increase mortality and morbidity (1). Malnutrition in these patients reduces quality of life, increases the risk of infection, increases the risk of diseases, and impairs wound healing. It also results in poor rehabilitation, fatigue, lethargy, as well as increased hospitalization and mortality in these patients (3). Therefore, it is essential to assess nutritional status for early diagnosis of malnutrition in these patients in order to take effective measures to initiate supportive nutritional program. The subjective global assessment (SGA) is a practical tool for assessment of nutritional status in hemodialysis patients (10). This technique assesses malnutrition based on medical history and physical examination. It is a high-speed, inexpensive, and easily reproducible tool that does not require specific training. It serves as a reliable screening method for malnutrition $(11,12)$.

Nutritional status was reported to differ in various races and regions. Therefore, it is essential to assess nutritional status of these patients (13).

\section{Objectives}

Given the high number of ESRD patients on hemodialysis in Ahvaz and the importance of mortality and morbidity in these patients, the present study aimed to assess the prevalence of malnutrition and some effective factors in the hemodialysis patients visiting educational treatment centers in Ahvaz.

\section{Patients and Methods \\ Study design}

This was a descriptive, analytical epidemiological study. The statistical population consisted of all chronic renal failure patients undergoing hemodialysis at treatment centers in three educational hospitals in Ahvaz in 2019. Inclusion criteria were older than 18 years old and at least 3 months after hemodialysis initiation. Informed written consent forms were obtained from the patients. Exclusion criteria were intact parathyroid hormone $(\mathrm{PTH})>800 \mathrm{pg} / \mathrm{mL}$, concomitant malignancy, infectious disease and severe sepsis during the last month, recipients of immunosuppressive drug, total parenteral nutrition, hepatitis $\mathrm{B}$ and $\mathrm{C}$, alcohol consumption, changes in dialysis technique in the last month, recipients of dietary supplements and amino acids except folic acid. Objective and method of the research were explained to the patients. Age, gender, body mass index (BMI), weekly dialysis duration and dialysis adequacy $(\mathrm{Kt} / \mathrm{V})$ were measured and collected. The patients' dry weight was measured after dialysis with the least clothing at two consecutive times using a scale (0.1 kg precision). Their heights were measured at standing posture barefoot while heels, buttocks, shoulders and head back were equally pressed against the wall $(0.5 \mathrm{~cm}$ precision $)$. BMI was calculated by dividing weight $(\mathrm{kg})$ by the square of height $\left(\mathrm{m}^{2}\right)$. The patients were categorized in three categories: $\mathrm{BMI}<18.5$ $\mathrm{kg} / \mathrm{m}^{2}, 18.5 \mathrm{~kg} / \mathrm{m}^{2}<\mathrm{BMI}<25 \mathrm{~kg} / \mathrm{m}^{2}$, and $\mathrm{BMI}>25 \mathrm{~kg} /$ $\mathrm{m}^{2}$. Dialysis adequacy was calculated based on percent reduction of urea (PRU) using the formula $\mathrm{Kt} / \mathrm{V}=(0.026$ $\times \mathrm{PRU})-0.46$. Minimum $\mathrm{Kt} / \mathrm{V}$ is 1.2 and $\mathrm{Kt} / \mathrm{V} \geq 1.2$ is acceptable. Blood specimens were taken from the patients prior to dialysis to test biochemical factors (serum albumin concentration, triglyceride and C-reactive protein [CRP]). Subjective global assessment was used to assess malnutrition status. It consists of two parts of medical history and physical examination. The first part assesses changes in weight in the past 6 months, diet, apparent gastrointestinal symptoms, functional capacity and hypercatabolic diseases (e.g. physical injuries, burns, inflammatory diseases, infection and malignant tumors). The second part evaluates loss of subcutaneous fat, muscle wasting, and edema. Score A is allocated to the patient with normal cases of the above factors. Score B is allocated to patients with mild to moderate cases of the above factors. Score $\mathrm{C}$ is given to patients with severe cases of the above factors. Score A represents normal nutrition, score B shows mild to moderate malnutrition, and score $\mathrm{C}$ denotes severe malnutrition in final scoring of SGA (14).

\section{Ethical issues}

The research followed the tenets of the Declaration of Helsinki. Written informed consent was obtained from all the patients. This study was approved by the Ethics Committee of Jundishapur University of Medical Sciences (Ethical code: IR.Ajums.REC.1397.951). Ethical issues and data confidentiality were observed in the course of project. Besides, this study was extracted from the residential thesis of Soudabeh Sabzali at this University.

\section{Statistical analysis}

Data analysis was performed using SPSS v. 22. Descriptive statistics (mean, standard deviation, frequency and percentage of frequency) was also used for data analysis. Kruskal-Wallis test, chi-square, Spearman's correlation, and logistic regression were also used for data analysis and assessment of relationship of each variable with nutritional status. The significance level was less than 0.05 .

Results

The participants consisted of 239 hemodialysis patients with $57.05 \pm 15.58$ (from 18 to 90 years old). Demographic data is shown in Table 1. Average BMI was $25.19 \pm 5.55$ $\mathrm{kg} / \mathrm{m}^{2}$ (from 14 to 53 ) and average Kt/V was $1.25 \pm 0.46$.

SGA scoring showed that 168 patients $(70.3 \%)$ had normal nutrition, 45 patients $(18.8 \%)$ had mild to moderate malnutrition and 26 patients $(10.9 \%)$ had severe malnutrition.

The relationships of each variable with nutritional status of hemodialysis patients are presented in Table 2. Age and CRP had a direct significant relationship with malnutrition 
Table 1. Demographic data in hemodialysis patients

\begin{tabular}{|c|c|c|}
\hline Variable & Group & No. (\%) \\
\hline \multirow{2}{*}{ Age $(y)$} & $\geq 65$ & 81 (33.9) \\
\hline & $<65$ & $158(66.1)$ \\
\hline \multirow{2}{*}{ Gender } & Male & $162(67.8)$ \\
\hline & Female & $77(32.2)$ \\
\hline \multirow{3}{*}{ BMI $\left(\mathrm{kg} / \mathrm{m}^{2}\right)$} & $\leq 18.5$ & $21(8.8)$ \\
\hline & $18.5-24.9$ & $119(49.8)$ \\
\hline & $>25$ & 99 (41.4) \\
\hline \multirow{2}{*}{ Dialysis duration (hours per week) } & $\leq 9$ & $229(95.8)$ \\
\hline & $>9$ & $10(4.2)$ \\
\hline \multirow{5}{*}{ C-Reactive protein (positive/negative) } & - & $135(56.5)$ \\
\hline & + & $30(12.6)$ \\
\hline & ++ & $28(11.7)$ \\
\hline & +++ & $43(18.0)$ \\
\hline & ++++ & $3(1.3)$ \\
\hline \multirow{2}{*}{ Triglyceride* $(\mathrm{mg} / \mathrm{dL})$} & $>150$ & $24(60)$ \\
\hline & $<150$ & $16(40)$ \\
\hline \multirow{2}{*}{ Albumin (g/dL) } & $\geq 3.5$ & $209(87.4)$ \\
\hline & $<3.5$ & $30(12.6)$ \\
\hline \multirow{2}{*}{ Dialysis adequacy (Kt/V) } & $\geq 1.2$ & $114(47.7)$ \\
\hline & $<12$ & $125(52.3)$ \\
\hline
\end{tabular}

*Triglyceride was only measured in 40 patients at Imam Khomeini hospital.
$(P<0.05)$. Serum albumin level and BMI had a statistically significant and inverse relationship with malnutrition $(P<0.05)$. Malnutrition was significantly more severe in women than men $(P<0.05)$. Kt/V and number of dialysis hours per week had no significant relationship with malnutrition $(P>0.05)$. Prevalence of malnutrition did not significantly differ in various hospitals.

Results of logistic regression model for predicting malnutrition in hemodialysis patients by different variables are shown in Table 3. BMI was the most effective factor in predicting varying degrees of malnutrition $(P=0.001)$. Gender and serum albumin are other effective factors in predicting severe malnutrition in hemodialysis patients $(P=0.001)$.

\section{Discussion}

The results of the study showed that most patients (70.3\%) had normal nutritional status. Varying degrees of malnutrition was also detected in $29.7 \%$ of hemodialysis patients $(18.8 \%$ mild malnutrition and $10.9 \%$ severe malnutrition).

Various studies have assessed malnutrition status in hemodialysis patients in different centers using SGA.

Yigit et al assessed nutritional status of hemodialysis patients with SGA and reported $36.4 \%$ of their patients had mild to moderate malnutrition since $23.6 \%$ severe malnutrition (7). Morais et al (15) assessed nutritional

Table 2. Relationship of studied variables with SGA score of hemodialysis patients

\begin{tabular}{|c|c|c|c|c|c|}
\hline Variable & Group & $\begin{array}{c}\text { Normal nutritional } \\
\text { status }\end{array}$ & $\begin{array}{l}\text { Mild to moderate } \\
\text { malnutrition }\end{array}$ & $\begin{array}{c}\text { Severe } \\
\text { malnutrition }\end{array}$ & P value** \\
\hline \multirow{2}{*}{ Age (y) } & & $48(59.3)$ & $22(27.2)$ & $11(13.6)$ & \multirow{2}{*}{0.024} \\
\hline & & 120 (75.9) & $23(14.6)$ & $15(9.5)$ & \\
\hline \multirow{2}{*}{ Gender } & Male & $119(73.5)$ & $32(19.8)$ & $11(6.8)$ & \multirow{2}{*}{0.013} \\
\hline & Female & $49(63.6)$ & $13(16.9)$ & $15(19.5)$ & \\
\hline \multirow{3}{*}{$\mathrm{BMI}\left(\mathrm{kg} / \mathrm{m}^{2}\right)$} & $\leq 18.5$ & $1(4.8)$ & $5(23.8)$ & $15(71.4)$ & \multirow{3}{*}{0.001} \\
\hline & $18.5-24.9$ & $77(64.7)$ & $32(26.9)$ & $10(8.4)$ & \\
\hline & $>25$ & 90 (90.9) & $8(8.1)$ & $1(1.0)$ & \\
\hline \multirow{2}{*}{ Dialysis duration (h/wk) } & $\geq 9$ & $161(70.3)$ & $44(19.2)$ & $24(10.5)$ & \multirow{7}{*}{0.047} \\
\hline & $<9$ & $7(70.0)$ & $1(10.0)$ & $2(20.0)$ & \\
\hline \multirow{5}{*}{$\begin{array}{l}\text { C-reactive protein (positive/ } \\
\text { negative) }\end{array}$} & - & $99(73.3)$ & $28(20.7)$ & $8(5.9)$ & \\
\hline & + & $19(63.3)$ & 5 (16.7) & $6(20.0)$ & \\
\hline & ++ & 19 (67.9) & $7(25.0)$ & $2(7.1)$ & \\
\hline & +++ & $28(65.1)$ & $5(11.6)$ & $10(23.3)$ & \\
\hline & ++++ & $3(100)$ & $0(0)$ & $0(0)$ & \\
\hline \multirow{2}{*}{ Triglyceride* (mg/dL) } & $>150$ & $16(66.7)$ & $5(20.8)$ & $3(12.5)$ & \multirow{2}{*}{0.796} \\
\hline & $<150$ & $11(68.8)$ & $4(25.0)$ & $1(6.3)$ & \\
\hline \multirow{2}{*}{ Albumin (g/dL) } & $\geq 3.5$ & $154(73.7)$ & $36(17.2)$ & $19(9.1)$ & \multirow{2}{*}{0.007} \\
\hline & $<3.5$ & $14(46.7)$ & $9(30.0)$ & $7(23.3)$ & \\
\hline \multirow{2}{*}{ Dialysis adequacy (Kt/V) } & $<1.2$ & $92(73.6)$ & $22(17.6)$ & $11(8.8)$ & \multirow{2}{*}{0.436} \\
\hline & $\geq 1.2$ & $76(66.7)$ & $23(20.2)$ & $15(13.2)$ & \\
\hline \multirow{3}{*}{ Hospital } & Imam Khomeini & $44(66.7)$ & $14(21.2)$ & $8(12.1)$ & \multirow{3}{*}{0.874} \\
\hline & Golestan & $54(74.0)$ & $13(17.8)$ & $6(8.2)$ & \\
\hline & Razi & $70(70.0)$ & $18(18.0)$ & $12(12.0)$ & \\
\hline
\end{tabular}

*Triglyceride was just measured in 40 patients.

** Values less than 0.05 are significant. 
Table 3. Results of logistic regression model in predicting malnutrition in hemodialysis patients

\begin{tabular}{|c|c|c|c|c|c|}
\hline \multirow{2}{*}{ Malnutrition status } & \multirow{2}{*}{ Variable } & \multirow{2}{*}{$P$ value } & \multirow{2}{*}{ Hazard ratio } & \multicolumn{2}{|c|}{$95 \% \mathrm{Cl}$ for hazard ratio } \\
\hline & & & & Lower limit & Upper limit \\
\hline \multirow{7}{*}{$\begin{array}{l}\mathrm{B} \text { (mild to moderate } \\
\text { malnutrition }\end{array}$} & Gender & 0.375 & 1.470 & 0.674 & 3.339 \\
\hline & Age (year) & 0.058 & 0.493 & 0.238 & 1.024 \\
\hline & Albumin (g/dL) & 0.062 & 2.615 & 0.954 & 7.164 \\
\hline & Dialysis duration (h/wk) & 0.258 & 0.271 & 0.028 & 2.601 \\
\hline & BMI $\left(\mathrm{kg} / \mathrm{m}^{2}\right)$ & 0.0001 & 0.818 & 0.742 & 0.901 \\
\hline & C-reactive protein (positive/negative) & 0.279 & 1.529 & 0.709 & 3.297 \\
\hline & Dialysis adequacy (Kt/V) & 0.817 & 1.146 & 0.361 & 3.633 \\
\hline \multirow{7}{*}{ C (severe malnutrition) } & Gender & 0.0001 & 9.818 & 2.735 & 35.243 \\
\hline & Age (year) & 0.549 & 0.691 & 0.206 & 2.317 \\
\hline & Albumin (g/dL) & 0.035 & 4.749 & 1.117 & 20.198 \\
\hline & Dialysis duration (h/wk) & 0.643 & 1.713 & 0.175 & 16.738 \\
\hline & BMI $\left(\mathrm{kg} / \mathrm{m}^{2}\right)$ & 0.0001 & 0.670 & 0.573 & 0.783 \\
\hline & C-reactive protein (Positive/Negative) & 0.577 & 0.708 & 0.22 & 2.377 \\
\hline & Dialysis adequacy (Kt/V) & 0.997 & 0.997 & 0.241 & 4.125 \\
\hline
\end{tabular}

* Reference value is A score.

status of 44 hemodialysis patients with SGA in Brazil and showed that $90.9 \%$ of the patients suffered from mild to moderate malnutrition and $4.6 \%$ suffered from severe malnutrition. These rates were higher than the rates reported in our study. Another study in Brazil showed $4.22 \%$ mild to moderate malnutrition in their hemodialysis patients (12).

The prevalence rates of malnutrition in hemodialysis patients in Spain (9), Jordan (19), Saudi Arabia (16), Iraq (17) and Palestine (18) were respectively as $41.1 \%, 62 \%$, $55 \%, 63.5 \%$ and $47.2 \%$. These rates were higher than the rates reported in this study. Differences in prevalence of malnutrition may be due to different environments, diets and treatment methods.

Gender was an effective factor in predicting severe malnutrition in hemodialysis patients in this study. Malnutrition was significantly higher in women than men. Another study carried out in Jordan (19) reported a significantly higher prevalence of malnutrition in women than men. These studies show the necessity of assessment of nutritional status in women undergoing hemodialysis, however Yigit et al in Turkey (7) found no relationship between gender and malnutrition in hemodialysis patients.

A direct and significant relationship was found between age and malnutrition in hemodialysis patients in this study. Many other studies have also reported that older patients tend to be more prone to nutritional deficiency than younger patients $(7,11,12,15)$. These results indicate the necessity of assessment of nutritional status in older patients.

The results of this study showed that BMI is the most effective factor in predicting varying degrees of malnutrition in hemodialysis patients. BMI had a statistically significant and inverse relationship with malnutrition status in this study. These results are consistent with the results of other studies. BMI is known as a nutritional marker $(7,12,15,20) . \mathrm{BMI}<20 \mathrm{~kg} / \mathrm{m}^{2}$ is an indicator of malnutrition (21), which was reported in $48.5 \%$ of patients with malnutrition. A meta-analysis showed lower mortality rates in hemodialysis patients with higher BMI (22).

CRP had a direct and significant relationship with malnutrition in this study. Serum albumin level had a significant and inverse relationship with malnutrition. It also predicts severe malnutrition in hemodialysis patients. However, in our study low-serum albumin level $(<3.5 \mathrm{~g} / \mathrm{dL})$ was measured in a small number of patients (12.6\%). Many other studies also showed a significant and inverse relationship between serum albumin level and malnutrition $(7,15,23)$. Rezeq et al also found no significant relationship between serum albumin level and malnutrition status (18). The difference might be due to effect of such other factors as proteinuria, a common problem in patients with renal failure. Dialysis can also lower plasma albumin levels. Since albumin is an acutephase reactant, most hemodialysis patients suffer from varying degrees of vascular inflammation. Therefore, albumin level changes in these patients. Hydration and acidemia also affect serum albumin level. However, the results of the present study and other similar studies showed that serum albumin level is a nutritional marker. Therefore, it is necessary to measure serum albumin level to evaluate clinical status (comorbidities, degrees of proteinuria, acid-base status and dialysis quality) and nutrition of hemodialysis patients (24). Specificity of 
albumin level as a nutritional marker decreases in case of inflammation and fluid overload (7). Inflammation is an effective factor in malnutrition often reported in hemodialysis patients (7). Many past studies have also reported a direct and significant relationship between malnutrition and inflammation (CRP serum level) (7).

The relationships of nutritional status with dialysis hours per week, triglyceride and Kt/V were not significant in this study. Likewise, Yigit et al in Turkey found no significant relationship between malnutrition and dialysis adequacy $(\mathrm{Kt} / \mathrm{V})$. The results of a recent study showed that lowdialysis adequacy $(\mathrm{Kt} / \mathrm{V})$ is associated with malnutrition in hemodialysis patients (12). A suitable dialysis dose can also improve malnutrition status in hemodialysis patients (25).

The difference in the prevalence of malnutrition and its associated factors in different studies can be due to differences in malnutrition assessment methods, age, duration of disease, comorbidities, inflammation, medications and supplements that affect nutritional status and associated factors. The difference might also be due to socioeconomic factors (e.g. literacy, knowledge and awareness of patients on renal failure and family support). Access to food, food habits and culture in each region are also involved in nutrition status of these patients.

\section{Conclusion}

Our study showed that the prevalence of malnutrition in hemodialysis patients was lower than the studies carried out in other countries based on SGA. High prevalence of severe malnutrition also necessitates periodic assessment and monitoring of nutrition in these patients. Comprehensive clinical nutrition services and counseling should be included in treatment program of these patients, especially in older people with lower BMI. Laboratory measurement of albumin serum, BMI and SGA can also help to assess nutritional status of patients in a cost-effective, inexpensive and simple manner in order to treat malnutrition as soon as possible.

\section{Limitations of the study}

Limitations of the study were absence of assessment of inflammatory markers (except for CRP) for determination of systematic inflammation, duration of disease and dialysis duration. The impact of comorbidities, calorie intake, socioeconomic status and income on malnutrition was not also assessed in this study. Therefore, it is recommended to carry out prospective studies to assess malnutrition and its associated factors, and the impact of preventive and therapeutic interventions on malnutrition status.

\section{Authors' contribution}

AG, FH and MK conducted the research. SS conducted the statistical analysis. SS prepared the primary draft. AG revised and prepared the final manuscript. All authors read and approved the final paper.

\section{Conflicts of interest}

The authors declare no conflicts of interest.

\section{Ethical considerations}

Ethical issues (including plagiarism, data fabrication, double publication) have been completely observed by the authors.

\section{Funding/Support}

This study was extracted from the research proposal with code number CRD-9704 and IR.AJUMS.REC.1397.951 approved on by the research deputy of Ahvaz Jundishapur University of Medical Sciences.

\section{References}

1. Zha Y, Qian Q. Protein Nutrition and Malnutrition in CKD and ESRD. Nutrients. 2017;9(3):208. doi:10.3390/ nu9030208

2. De Nicola L, Zoccali C. Chronic kidney disease prevalence in the general population: Heterogeneity and concerns. Nephrol Dial Transplant. 2016;31:331-5.

3. Ortiz A, Covic A, Fliser D, Fouque D, Goldsmith D, Kanbay M, et al. Epidemiology, contributors to, and clinical trials of mortality risk in chronic kidney failure. Lancet. 2014;383:1831-43.

4. Robinson BM, Zhang J, Morgenstern H, Bradbury BD, Ng LJ, McCullough KP, et al. Worldwide, mortality risk is high soon after initiation of hemodialysis. Kidney Int. 2014;85: 158-65. doi: 10.1038/ki.2013.252.

5. Eckardt KU, Gillespie IA, Kronenberg F, Richards S, Stenvinkel P, Anker SD, et al. High cardiovascular event rates occur within the first weeks of starting hemodialysis. Kidney Int. 2015;88:1117-25. doi: 10.1038/ki.2015.117.

6. Wang AY, Lam CW, Chan IH, Wang M, Lui SF, Sanderson JE. Sudden cardiac death in end-stage renal disease patients: A 5-year prospective analysis. Hypertension. 2010;56:210-6.

7. Yigit IP, Ulu R, Celiker H, Dogukan A. Evaluation of nutritional status using anthropometric measurements and MQSGA in geriatric hemodialysis patients. Northern clinics of Istanbul. 2016;3:124-130.

8. Becker PJ, Nieman Carney L, Corkins MR, Monczka J, Smith E, Smith SE, et al. Consensus statement of the Academy of Nutrition and Dietetics/American Society for Parenteral and Enteral Nutrition: indicators recommended for the identification and documentation of pediatric malnutrition (undernutrition). J Acad Nutr Diet. 2014;114:1988-2000. doi: $10.1016 /$ j.jand.2014.08.026

9. Gracia-Iguacel C, González-Parra E, Pérez-Gómez MV, Mahíllo I, Egido J, Ortiz A, et al. Prevalence of proteinenergy wasting syndrome and its association with mortality in haemodialysis patients in a center in Spain. Nefrologia. 2013;33:495-505.

10. Chung S, Koh ES, Shin SJ, Park CW. Malnutrition in patients with chronic kidney disease. Open J Int Med. 2012; 2:89-99.

11. Yamada K, Furuya R, Takita T, Maruyama Y, Yamaguchi Y, Ohkawa S, et al. Simplified nutritional screening tools for patients on maintenance hemodialysis. Am J Clin Nutr. 
2008;87:106-13. doi: 10.1093/ajcn/87.1.106

12. Freitas ATB, Vaz IMF, Filizola IM, Ferraz SF, Peixoto MRG, Campos MIVM. Prevalence of malnutrition and associated factors in hemodialysis patients. Revista de Nutrição. 2014; 27: 357-366.

13. Noori N, Kovesdy CP, Dukkipati R, Feroze U, Molnar MZ, Bross R, et al. Racial and ethnic differences in mortality of hemodialysis patients: Role of dietary and nutritional status and inflammation. Am J Nephrol. 2011;33:157-67.doi: 10.1159/000323972.

14. Barker LA, Gout BS, Crowe TC. Hospital malnutrition: prevalence, identification and impact on patients and the healthcare system. Int J Environ Res Public Health. 2011;8:514-27. doi: 10.3390/ijerph8020514.

15. Morais AA, Silva MA, Faintuch J, Vidigal EJ, Costa RA, Lyrio DC, et al. Correlation of nutritional status and food intake in hemodialysis patients. Clinics (Sao Paulo). 2005;60:185-92.

16. Alharbi K, Enrione EB. Malnutrition is prevalent among hemodialysis patients in Jeddah, Saudi Arabia. Saudi J Kidney Dis Transpl. 2012;23:598-608.

17. Al-Saedy AJ, Al-Kahichy HR. The current status of hemodialysis in Baghdad. Saudi J Kidney Dis Transpl. 2011;22:362-7.

18. Rezeq HA, Khdair LN, Hamdan ZI, Sweileh WM. Prevalence of malnutrition in hemodialysis patients: A single-center study in Palestine. Saudi J Kidney Dis Transpl. 2018;29:332-40.

19. Tayyem RF, Mrayyan MT, Heath DD, Bawadi HA.
Assessment of nutritional status among ESRD patients in Jordanian hospitals. J Ren Nutr. 2008;18:281-7.

20. Chen J, Peng H, Yuan Z, Zhang K, Xiao L, Huang J, et al. Combination with anthropometric measurements and MQSGA to assess nutritional status in Chinese hemodialysis population. Int J Med Sci. 2013;10:974-80. doi: $\quad$ 10.7150/ijms.5811.

21. Fouque D, Vennegoor M, ter Wee P, Wanner C, Basci A, Canaud B, et al. EBPG guideline on nutrition. Nephrol Dial Transplant. 2007;22 Suppl 2:45-87.

22. Jialin W, Yi Z, Weijie Y. Relationship between body mass index and mortality in hemodialysis patients: a metaanalysis. Nephron Clin Pract. 2012;121:102-11. doi: $10.1159 / 000345159$.

23. Mikolasevic I, Lukenda V, Racki S, Milic S, SladojeMartinovic B, Orlic L. Nonalcoholic fatty liver disease (nAFlD) - a new factor that interplays between inflammation, malnutrition, and atherosclerosis in elderly hemodialysis patients. Clin Interv Aging. 2014;9:1295-303. doi: $\quad$ 10.2147/CIA.S65382.

24. Kaysen GA, Dubin JA, Muller HG, Rosales L, Levin NW, Mitch WE, et al. Inflammation and reduced albumin synthesis associated with stable decline in serum albumin in hemodialysis patients. Kidney Int. 2004;65:1408-15.

25. Azar AT, Wahba K, Mohamed AS, Massoud WA. Association between dialysis dose improvement and nutritional status among hemodialysis patients. Am J Nephrol. 2007; 27:1139. doi: 10.1159/000099836.

Copyright $\odot 2020$ The Author(s); Published by Nickan Research Institute. This is an open-access article distributed under the terms of the Creative Commons Attribution License (http://creativecommons.org/licenses/by/4.0), which permits unrestricted use, distribution, and reproduction in any medium, provided the original work is properly cited. 